\title{
A Retrospective Analysis of Acute Appendicitis in Children Treated with Laparoscopic Appendectomies at a Single Center in Dubai, UAE
}

\author{
Saad Syed ${ }^{a}$ Hussein Naji ${ }^{a, b}$ \\ aCollege of Medicine, Mohammed Bin Rashid University of Medicine and Health Sciences, Dubai, UAE; \\ ${ }^{b}$ Department of Pediatric Surgery, Mediclinic Parkview Hospital, Dubai, UAE
}

\section{Keywords}

Acute appendicitis · Children · Pediatric · Laparoscopic appendectomy

\begin{abstract}
Background: Appendicitis is one of the most common emergency conditions that occur in the pediatric population. The condition is usually suspected clinically, and the diagnosis is confirmed by radiological investigations such as ultrasound, CT scan, or MRI. This study was conducted to contribute to global databases by presenting data from the Middle East with an objective of identifying the clinical characteristics of children who were diagnosed with acute appendicitis and underwent laparoscopic appendectomy at a single pediatric surgery center in Dubai. Methods: A 2-year hospital-based retrospective cohort study was conducted at Mediclinic Parkview Hospital, Dubai, enrolling all patients younger than 14 years who were diagnosed with acute appendicitis and who underwent laparoscopic appendectomy. Demographic data, clinical presentation, laboratory, radiological and pathology findings, postoperative complications, and readmission rates were analyzed. Results: Fifty-six patients were operated on and enrolled in this study. All patients (56/56) presented with abdominal pain, while an associated fever was
\end{abstract}

present in $44.6 \%(25 / 56) ; 78.5 \%(44 / 56)$ of the patients had nausea but $64.3 \%(36 / 56)$ had vomiting. On examination, all patients had abdominal tenderness, while rebound tenderness in the right iliac fossa (RIF) was found in $92.8 \%(52 / 56)$ of the patients. Laboratory investigations showed elevated leukocyte count in $76.7 \%$ (43/56) of patients. Appendicitis was diagnosed on ultrasound in $57.4 \%$ (31/54) of the patients, and free fluid was visualized in $40.7 \%$ (22/54) of the patients. Appendicitis was diagnosed on CT scan in all 25 patients, and free fluid was visualized in 64\% (16/25) of the patients. The number of admission days ranged from 1 to 5 days, with a median of 2 days. And 62.5\% (35/56) of patients were discharged in the first 2 days from admission. Major and minor complications after surgery were documented in 4 and 14 patients, respectively. Pathology reports showed features of acute appendicitis in all the resected appendices. Conclusions: Acute appendicitis in children should be suspected in all children with acute abdominal pain. This study can help guide the management of pediatric appendicitis and allow proper and standardized documentation of findings and judicious use of laboratory and radiological investigations.

(C) 2021 The Author(s)

Published by S. Karger AG, Basel karger@karger.com www.karger.com/dmj

Karger $\stackrel{\text { ' }}{5}$

GOPEN ACCESS
(C) 2021 The Author(s)

Published by S. Karger AG, Basel

This is an Open Access article licensed under the Creative Commons Attribution-NonCommercial-4.0 International License (CC BY-NC) (http://www.karger.com/Services/OpenAccessLicense), applicable to the online version of the article only. Usage and distribution for commercial purposes requires written permission.
Correspondence to:

Saad Syed, saad.syed@ @students.mbru.ac.ae 


\section{Introduction}

Appendicitis is one of the most common reasons for presentation to the emergency department in the pediatric population, with a lifetime risk of $8.6 \%$ in male and $6.7 \%$ in female individuals, occurring usually in the second decade of life, with a slight predominance in male individuals [1-3]. Appendicitis was regarded as the fifth most common reason for hospital admission in the pediatric population in the USA $(97.4$ stays per 100,000$)$ [4]. The incidence of acute pediatric appendicitis in the 21st century ranges between 100 and 151 cases per 100,000 person-years depending on geographical location [5].

Patients are usually diagnosed clinically; however, a variety of scores have been proposed to aid in the process of diagnosis [6-9]. Additionally, laboratory investigations such as a complete blood count, absolute neutrophil count, and C-reactive protein (CRP) levels are often ordered. Radiological investigations such as ultrasound, CT scan, and MR imaging are also ordered to confirm the diagnosis of appendicitis. Although most pediatric patients who present with appendicitis are managed with appendectomy, nonoperative management of pediatric appendicitis is being discussed as an alternate option in management $[2,3,10]$.

Although a lot of research articles have been published on the topic of appendicitis, we aimed to contribute to the global databases by presenting a research study from the Middle East. The objective of this study is to identify the clinical characteristics and laboratory findings of children with acute appendicitis at a single hospital in Dubai.

\section{Materials and Methods}

A 2-year retrospective descriptive cohort study was conducted at Mediclinic Parkview Hospital, Dubai, on all children younger than 14 years who were diagnosed with acute appendicitis. The research was conducted ethically in accordance with the World Medical Association Declaration of Helsinki and was approved by the Mediclinic Middle East Research and Ethics Committee (MCME.CR 150. MPAR. 2020) and Dubai Scientific Research Ethics Committee (DSREC), DHA (DSREC-11/2020_18). De-identification of patients' data used in this research was carried out retrospectively, and the need to obtain a written consent, rather than the general patient's consent to treat and use their data for research, was waived by the MCME. A total population sampling method was used to sample the population.

\section{Inclusion Criteria}

All children younger than 14 years who were diagnosed with acute appendicitis and treated surgically with laparoscopic appendectomy at Mediclinic Parkview Hospital from September 2018 to August 2020 were included in this study.

\section{Exclusion Criteria}

Children with acute appendicitis (8 patients) who were treated with only medical treatment using antibiotics during the peak of the COVID-19 pandemic outbreak were excluded from the study as they were not operated on because of the restrictions to prevent the spread of the pandemic [3]. The analyzed variables were age, gender, presenting symptoms (including abdominal pain, fever, nausea, vomiting, and diarrhea), signs (including abdominal tenderness, guarding, rebound tenderness, and dehydration), significant past medical history, laboratory investigations (including complete blood count, CRP levels, and urinalysis), radiological investigations, number of admission days, postoperative complications, presence of perforation, peritonitis, pathology findings, and readmission rates. The statistical tool used to analyze the data was Microsoft Excel.

\section{Results}

Fifty-six patients were operated on during this 2-year period and enrolled in the study. Twenty-one (37.5\%) patients were female, and $35(62.5 \%)$ patients were male. The ages of the patients ranged from 5 to 14 years, with a mean (SD) age of 9 (4.24) years. Six (10.7\%) patients had chronic comorbidities: 2 (3.5\%) patients had asthma, 2 (3.5\%) patients had familial Mediterranean fever, 1 (1.7\%) patient had chronic anemia, and 1 (1.7\%) patient had type 1 diabetes. Another 2 (3.5\%) patients had a history of orchiopexy procedure earlier in their life.

All patients $(56 / 56)$ presented with abdominal pain, while an associated fever was present in $44.6 \%(25 / 56)$; $78.5 \%(44 / 56)$ of the patients had nausea but $64.3 \%$ (36/56) had vomiting. Diarrhea was documented in $12.5 \%$ (7/56) of the patients. On examination, all patients had abdominal tenderness, while rebound tenderness in the right iliac fossa (RIF) was found in $92.8 \%(52 / 56)$ of the patients. Guarding was found in $32.1 \%(18 / 56)$ of the patients. Dehydration was documented in $37.5 \%(21 / 56)$ of the patients. Laboratory investigations showed elevated leukocyte count $(>11,000$ cells $/ \mu \mathrm{L})$ in $76.7 \%(43 / 56)$ of patients. An elevated neutrophil count was considered to be $>8,500$ cells/ $\mu \mathrm{L}$ and was found in $70.4 \%(38 / 54)$ of the patients. An elevated CRP level was found in $57.1 \%$ $(32 / 56)$ of patients. Culture results from a swab taken during surgery from the surface of the appendix showed a growth of bacteria in $48.2 \%(27 / 56)$ of patients. E. coli was the predominant isolated bacteria (24 patients), while Pseudomonas was isolated in 7 patients (6 of them had growth of E. coli as well). The following types of bacteria were detected as well: Klebsiella pneumoniae (1 patient), Staphylococcus aureus (1 patient), Streptococcus gordonii (1 patient), and Streptococcus anginosus (1 patient). Uri- 
nalysis results were not available in the records of $17 \mathrm{pa}$ tients. A normal urinalysis was observed in $28.2 \%(11 / 39)$ of the patients. Erythrocytes were found in 15.4\% (6/39) of the patients, leukocytes were found in $7.7 \%(3 / 39)$ of the patients, ketones were found in $53.8 \%(21 / 39)$ of the patients, proteins were found in $17.9 \%$ (7/39) of the patients, leukocyte esterase were found in $12.8 \%(5 / 39)$ of the patients, and hemoglobin, urobilinogen, and epithelial cells were found in $2.56 \%(1 / 39)$ of the patients. Ultrasound scan of the abdomen and pelvis was performed for $96.4 \%$ (54/56) of the patients. Appendicitis was diagnosed on ultrasound in $57.4 \%(31 / 54)$ of the patients, and free fluid was visualized in $40.7 \%(22 / 54)$ of the patients. CT scan of the abdomen and pelvis was performed to $44.6 \%$ $(25 / 56)$ of the patients. Appendicitis was diagnosed on CT scan in all 25 patients, and free fluid was visualized in $64 \%(16 / 25)$ of the patients. The number of admission days ranged from 1 to 5 days, with a median of 2 days; $62.5 \%(35 / 56)$ of patients were discharged in the first 2 days from admission. Only $2(3.6 \%)$ patients were discharged on day 5. Major complications after surgery were documented in 4 patients in the form of intra-abdominal collection of fluid that required additional IV antibiotic therapy. One of them developed sepsis and needed 5 days stay in hospital. Fourteen patients had minor complications after surgery: 5 patients developed mild erythema at the wound site, 3 patients developed fever, 2 patients developed vomiting, 1 patient developed shortness of patient, 1 patient had a suprapubic wound dehiscence, and 2 patients had a yellowish discharge from the sub-umbilical wound. One (1.8\%) patient needed to be readmitted to the hospital for additional IV antibiotic treatment post-surgery because of an abdominal abscess. All patients were successfully managed and eventually discharged from the hospital. Pathology reports showed features of acute appendicitis in all the resected appendices $(55 / 55)$. The appendix was perforated in $40 \%(22 / 55)$ of the patients, gangrenous appendix in $16.4 \%(10 / 55)$ of the patients, presence of eosinophilic infiltration in $1.8 \%$ (1/55) of the patients, and presence of intestinal worms in $1.8 \%(1 / 55)$ of the patients. One patient's pathology report was not available on the patient's electronic medical record. These results are summarized in Table 1.

\section{Discussion}

The most likely symptom and sign indicative of appendicitis in our study was abdominal pain and rebound tenderness, which were similar to the findings in the system-

Acute Appendicitis in Children Treated with Laparoscopic Appendectomy

Table 1. Summary of the symptoms and signs, laboratory findings, urinalysis findings, radiological findings, complications, and pathology report findings found in the study population

pathology report findings found in the study population

Patients,

$n / N(\%)$

Symptom or sign

Abdominal pain

Fever

Nausea

Vomiting

Diarrhea

Abdominal tenderness

Guarding on examination

Rebound tenderness

Dehydration

Laboratory investigation

Leukocyte count $>11,000$ cells $/ \mu \mathrm{L}$

Neutrophil count $>8,500$ cells $/ \mu \mathrm{L}$

CRP between 10 and $100 \mathrm{mg} / \mathrm{L}$

Growth of bacteria

Growth of E. coli

Growth of Pseudomonas

Growth of Klebsiella pneumoniae

Growth of Staphylococcus aureus

Growth of Streptococcus gondii

Growth of Streptococcus anginosus

Urinalysis investigation

RBCs present

WBCs present

Ketones present

Proteins present

Leukocyte esterase positive

Hemoglobin present

Urobilinogen present

Epithelial cells present

Normal urinalysis

Radiological investigation

Ultrasound was performed

Appendicitis diagnosed on ultrasound

Free fluid visualized on ultrasound

CT scan was performed

Appendicitis diagnosed on CT scan

Free fluid visualized on CT scan

Complication

No complications

Intra-abdominal abscess

Sepsis

Mild erythema at the wound site

Fever

Episodes of vomiting

Shortness of breath

Suprapubic wound dehiscence

Yellowish discharge

Readmission

Pathology report finding

Presence of acute appendicitis

Perforation of the appendix

Gangrenous appendix

Presence of eosinophilic infiltration

Presence of intestinal worms in the appendix

CRP, C-reactive protein.
$56 / 56(100)$

25/56 (44.6)

$44 / 56(78.5)$

$36 / 56(64.3)$

$7 / 56(12.5)$

$56 / 56(100)$

$18 / 56(32.1)$

$52 / 56(92.8)$

$21 / 56(37.5)$

$43 / 56(76.7)$

$38 / 54(70.4)$

$32 / 56(57.1)$

27/56 (48.2)

24/56 (42.8)

$7 / 56(12.5)$

$1 / 56(1.8)$

$1 / 56(1.8)$

$1 / 56(1.8)$

$1 / 56(1.8)$

$6 / 39(15.4)$

$3 / 39(7.7)$

21/39 (53.8)

$7 / 39(17.9)$

$5 / 39(12.8)$

$1 / 39(2.56)$

$1 / 39(2.56)$

$1 / 39(2.56)$

$17 / 39(28.2)$

$54 / 56(96.4)$

$31 / 54(57.4$

22/54 (40.7)

25/56 (44.6)

$25 / 25(100)$

$16 / 25$ (64)

$38 / 56(67.85)$

$3 / 56(5.35)$

$1 / 56(1.8)$

$5 / 56(8.9)$

$3 / 56(5.35)$

$2 / 56(3.6)$

$1 / 56(1.8)$

$1 / 56(1.8)$

$2 / 56(3.6)$

$1 / 56(1.8)$

$55 / 55(100)$

22/55 (40)

$9 / 55(16.4)$

$1 / 55(1.8)$

1/55 (1.8) 
atic review by Benabbas et al. [11]. In our study, different doctors documented abdominal pain as "diffuse abdominal pain, right lower quadrant pain, and abdominal pain radiating to the right lower quadrant" depending on physical examination findings and the ability to communicate with the patient. As such, we have considered all of these descriptions as abdominal pain suspicious of appendicitis. Similarly, we considered fever either to be reported by the parents in the history or by measuring the temperature of the patient at the hospital.

Although the Alvarado score and Pediatric Appendicitis Score are useful tools in excluding acute appendicitis in the pediatric population [12]; we relied on a high index of suspicion of acute appendicitis in children with abdominal pain. Thus, we have not used any particular score system.

According to certain scores and guidelines, it is recommended requesting routine laboratory tests and serum inflammatory biomarkers in order to predict the severity of the inflammation and the need for imaging investigation. In our study, complete blood count and CRP were routinely ordered for all patients with suspected acute appendicitis. Accordingly, radiological investigations were ordered to confirm the diagnosis of appendicitis.

Three modes of radiological imaging have been described in the literature for the diagnosis of appendicitis: ultrasound, CT scan, and MRI. One systematic review by Doria et al. [13] conducted in 2006 showed that CT imaging had a significantly higher sensitivity than ultrasound in the diagnosis of pediatric appendicitis. However, a recent study by Lee and Yun [14] demonstrated a sensitivity and specificity of $95 \%$ each using emergency physician point-of-care ultrasound in the diagnosis of pediatric appendicitis. The study by Kharbanda et al. [15] demonstrates that there is a strong negative correlation between ultrasound and cost and a strong positive correlation between CT rate and cost and suggest ultrasound as a primary imaging modality for the confirmation of the diagnosis of pediatric appendicitis.

The routine in our hospital is to perform ultrasound abdominal study as the primary modality of radiology investigation to diagnose appendicitis in children. This was applied to all our patients apart from 2 patients who were referred from another facility with a CT scan report confirming the diagnosis of acute appendicitis. Ultrasound has some known limitations: it requires specialized training, it is operator dependent, it may be affected by the body habitus of the patient, and it may be different in pediatric age-groups as compared to adult populations [15].
CT imaging has demonstrated sensitivities and specificities of $94-97 \%$ and $94-99 \%$ respectively. The advantages of CT imaging include rapid acquisition time and no operator dependency. The disadvantages of CT imaging are limited availability depending on geographical location, cost, and radiation exposure $[3,16,17]$. In collaboration with our radiology department, we tried to reduce the radiation to minimum and to limit the use of CT scans to those with equivocal ultrasound results.

MR imaging has demonstrated sensitivities and specificities of $96-97 \%$ and $96-98 \%$, respectively. The advantage of MR imaging is the lack of radiation exposure $[3,18,19]$. However, due to the disadvantages of limited availability, slow acquisition time, increased cost, and requirement of sedation of the patient, MRI scans are not performed for the diagnosis of pediatric appendicitis in our hospital.

There is some discussion about managing early probable cases of appendicitis nonoperatively using antibiotics and utilizing surgery only in clinically advanced cases. The advantages of this could be reduced number of CT scans and radiation exposure, decrease in perforation rates, negative appendectomy rates and overall appendectomy rates, and decrease in risks related to complications of anesthesia and surgery. The disadvantages include overtreatment with antibiotics, recurrence, and risk of progression of the disease process [20]. Svensson et al. [2] in their randomized controlled trial found that nonoperative management is as safe as initial treatment. However, the failure rate increases in the presence of appendicolith, and surgery is recommended in such cases $[2,12]$. In our hospital, all pediatric patients are managed surgically, and nonoperative management was only done for some patients during the COVID-19 pandemic, which were excluded from this study.

Studies show that there is no difference in complication rates whether patients underwent emergent or urgent appendectomy. Thus, patients presenting overnight were admitted into the hospital and operated on the next day to avoid stress on both parties [3].

The risk of appendiceal perforation is greater in children than in adults and is $7.7 \%$ in the first $24 \mathrm{~h}$, increasing linearly over time, and is associated more with pre-hospital delay, rather than in-hospital delay [21]. Other associated factors include the age of the child, race, social class, insurance status, and presence of appendicolith [22, 23]. Tan et al. [24] also found that an increased body temperature on admission increased the risk of appendiceal perforation.

Another factor to consider is that all pediatric appendectomies were performed by a pediatric surgeon in our 
hospital. Kim et al. [25] showed that patients operated by a pediatric surgeon have reduced operation times, reduced length of hospital stay, increased likelihood of laparoscopic operation, and less peritoneal drainage as compared to patients operated by a general surgeon. However, this luxury may not be available depending on geographical location.

\section{Limitations and Strengths}

There are a number of limitations identified by the authors in this study. First, the sample size of patients is small. Second, not all patients received the same diagnostic tests, resulting in variable numbers for different investigations conducted. There could be a selection bias as only patients successfully diagnosed by clinical diagnosis supported by radiological investigation were operated on. Thus, for the reasons of beneficence and nonmaleficence (doing the best for the patients for the lowest costs and less exposure to radiation, etc.) not all 56 patients underwent all tests such as CT scans and urinalysis. Third, there is an expertise bias in the utilization of ultrasound as different operators performed the test for different patients over the 2-year period. Due to the COVID-19 pandemic, some patients were treated nonoperatively, and these patients were excluded from this study. Also due to the pandemic, 2 patients did not have all their laboratory investigations documented in their electronic medical record and 1 patient did not have their pathology report documented into their electronic medical record. However, the strengths of this study are that all surgeries were performed by a single pediatric surgeon, providing standardization in care, and all cases were confirmed by pathology reports to be appendicitis.

\section{Conclusion}

Acute pediatric appendicitis is a clinical diagnosis based on patient history taking and physical examination findings, supported by laboratory investigations, and confirmed by radiological investigations such as ultrasound and CT scan. This study can help guide the man- agement of pediatric appendicitis and allow proper and standardized documentation of findings and judicious use of laboratory and radiological investigations.

\section{Acknowledgements}

We would like to thank Z.K. for her contributions in receiving ethical approval for the study from both Ethics Committees.

\section{Statement of Ethics}

The research was conducted ethically in accordance with the World Medical Association Declaration of Helsinki and was approved by the Mediclinic Middle East Research and Ethics Committee (MCME.CR 150. MPAR. 2020) and Dubai Scientific Research Ethics Committee (DSREC), DHA (DSREC-11/2020_18). De-identification of patients' data used in this research was carried retrospectively, and the need to obtain a written consent, rather than the general patient's consent to treat and use their data for research, was waived by the MCME.

\section{Conflict of Interest Statement}

The authors have no conflicts of interest to declare.

\section{Funding Sources}

This research received no external funding.

\section{Author Contributions}

Both authors contributed equally to the paper. S.S. drafted the study and collected the data. S.S. and H.N. drafted the manuscript. H.N. suggested the topic, supervised study, and reviewed the manuscript. All authors read and approved the final manuscript.

\section{Data Availability Statement}

The data that support the findings of this study are not publicly available due to confidentiality reasons but are available from correspondence author upon reasonable request.

\section{References}

Acute Appendicitis in Children Treated

with Laparoscopic Appendectomy
1 St. Peter SD, Sharp SW, Holcomb GW, Ostlie DJ. An evidence-based definition for perforated appendicitis derived from a prospective randomized trial. J Pediatr Surg. 2008;43(12): $2242-5$.

2 Svensson JF, Patkova B, Almström M, Naji H, Hall NJ, Eaton S, et al. Nonoperative treatment with antibiotics versus surgery for acute nonperforated appendicitis in children: a pi- lot randomized controlled trial. Ann Surg. 2015;261(1):67-71.

3 Rentea R, St. Peter S. Pediatric appendicitis. Surg Clin North Am. 2017;97(1):93-112.

4 Witt WP, Weiss AJ, Elixhauser A. Overview of hospital stays for children in the United States, 2012 \#187. 2020. https://hcup-us.ahrq. gov/reports/statbriefs/sb187-Hospital-StaysChildren-2012.jsp Dec 24. 
5 Ferris M, Quan S, Kaplan BS, Molodecky N, Ball CG, Chernoff GW, et al. The global incidence of appendicitis: a systematic review of population-based studies. Ann Surg. 2017; 266(2):237-41.

6 Alvarado A. A practical score for the early diagnosis of acute appendicitis. Ann Emerg Med. 1986;15(5):557-64.

7 Samuel M. Pediatric appendicitis score. J Pediatr Surg. 2002;37(6):877-81.

8 Chong CF, Adi MI, Thien A, Suyoi A, Mackie AJ, Tin AS, et al. Development of the RIPASA score: a new appendicitis scoring system for the diagnosis of acute appendicitis. Singapore Med J. 2010;51(3):220-5.

9 Andersson M, Andersson RE. The appendicitis inflammatory response score: a tool for the diagnosis of acute appendicitis that outperforms the alvarado score. World J Surg. 2008; 32(8): 1843-9.

10 Jaschinski T, Mosch C, Eikermann M, Neugebauer EA. Laparoscopic versus open appendectomy in patients with suspected appendicitis: a systematic review of meta-analyses of randomised controlled trials. BMC Gastroenterol. 2015;15(1):48.

11 Benabbas R, Hanna M, Shah J, Sinert R. Diagnostic accuracy of history, physical examination, laboratory tests, and point-of-care ultrasound for pediatric acute appendicitis in the emergency department: a systematic review and meta-analysis. Acad Emerg Med. 2017; 24(5):523-51.
12 Di Saverio S, Podda M, De Simone B, Ceresoli M, Augustin G, Gori A, et al. Diagnosis and treatment of acute appendicitis: 2020 update of the WSES Jerusalem guidelines. World J Emerg Surg. 2020;15(1):27.

13 Doria AS, Moineddin R, Kellenberger CJ, Epelman M, Beyene J, Schuh S, et al. US or CT for diagnosis of appendicitis in children and adults? A meta-analysis. Radiology. 2006; 241(1):83-94.

14 Lee SH, Yun SJ. Diagnostic performance of emergency physician-performed point-ofcare ultrasonography for acute appendicitis: a meta-analysis. Am J Emerg Med. 2019;37(4): 696-705.

15 Kharbanda AB, Christensen EW, Dudley NC, Bajaj L, Stevenson MD, Macias CG, et al. Economic analysis of diagnostic imaging in pediatric patients with suspected appendicitis. Acad Emerg Med. 2018;25(7):785-94.

16 Kim DW, Yoon HM, Lee JY, Kim JH, Jung AY, Lee JS, et al. Diagnostic performance of CT for pediatric patients with suspected appendicitis in various clinical settings: a systematic review and meta-analysis. Emerg Radiol. 2018;25(6):627-37.

17 Yoon HM, Suh CH, Cho YA, Kim JR, Lee JS, Jung AY, et al. The diagnostic performance of reduced-dose CT for suspected appendicitis in paediatric and adult patients: a systematic review and diagnostic meta-analysis. Eur Radiol. 2018;28(6):2537-48.

18 Moore MM, Kulaylat AN, Hollenbeak CS, Engbrecht BW, Dillman JR, Methratta ST. Magnetic resonance imaging in pediatric appendicitis: a systematic review. Pediatr Radiol. 2016;46(6):928-39.
19 Kim JR, Suh CH, Yoon HM, Jung AY, Lee JS $\mathrm{Kim} \mathrm{JH}$, et al. Performance of MRI for suspected appendicitis in pediatric patients and negative appendectomy rate: a systematic review and meta-analysis. J Magn Reson Imaging. 2018;47(3):767-78.

20 Johnson SM, Puapong DP, Peebles C, Ishihara K, Bogenberger K, Woo RK. A combined medical/surgical appendicitis pathway decreases pediatric CT utilization, perforation, and negative appendectomy rates. Ann Pediatr Surg. 2020;16(1): 10.

21 Narsule CK, Kahle EJ, Kim DS, Anderson AC, Luks FI. Effect of delay in presentation on rate of perforation in children with appendicitis. Am J Emerg Med. 2011;29(8):890-3.

22 Howell EC, Dubina ED, Lee SL. Perforation risk in pediatric appendicitis: assessment and management. Pediatric Health Med Ther. 2018;9:135-45.

23 Aneiros B, Cano I, García A, Yuste P, Ferrero E, Gómez A. Pediatric appendicitis: age does make a difference. Rev Paul Pediatr. 2019; 37(3):318-24.

24 Tan PH, Teng XX, Gan ZY, Tan SQ. A study on the clinical factors associated with acute appendicitis and perforated appendicitis among children in a Secondary Medical Centre in Malaysia. Malays J Med Sci. 2020;27(4): 139-46.

25 Kim Y, Jung K, Ryu YJ, Moon SB. Pediatric appendectomy: the outcome differences between pediatric surgeons and general surgeons. Surg Today. 2016;46(10):1181-6. 